\title{
Complete response to crizotinib in a metastatic adenocarcinoma of unknown primary harboring MET amplification and NTRKI co-occurring mutation
}

This article was published in the following Dove Press journal:

OncoTargets and Therapy

\author{
Jing-hao Yao' \\ An-sheng Wang ${ }^{2}$ \\ Xiao-jing Wang ${ }^{3,4}$ \\ Lu Zhang' \\ Yue Zhu' \\ Yu-rong $\mathrm{Ou}^{5}$ \\ Zi-shu Wang' \\ Yan Yang'
}

'Department of Medical Oncology, The First Affiliated Hospital of Bengbu Medical College, Bengbu 233004, People's Republic of China; ${ }^{2}$ Department of Thoracic Surgery, The First Affiliated Hospital of Bengbu Medical College, Bengbu 233004, People's Republic of China; ${ }^{3}$ Department of Respiration, The First Affiliated Hospital of Bengbu Medical College, Bengbu 233004, People's Republic of China; ${ }^{4}$ Anhui Clinical and Preclinical Key Laboratory of Respiratory Diseases, Bengbu 233004, People's Republic of China; ${ }^{5}$ Department of Pathology, The First Affiliated Hospital of Bengbu Medical College, Bengbu 233004, People's Republic of China
Correspondence: Yan Yang

Department of Medical Oncology, The First Affiliated Hospital of Bengbu Medical

College, Bengbu, Anhui 233004, People's

Republic of China

Tel/Fax +865523074480

Email qiannianhupo@I63.com

\begin{abstract}
Carcinomas of unknown primary (CUPs) have poor prognosis due to the paucity of data on their clinical characteristics and laboratory features, and empirical chemotherapy still remains the critical management for this kind of disease. This study aimed to present the knowledge of treating an elderly man with metastatic adenocarcinoma of unknown primary and also with a history of long-term hypertension and renal cysts. He was identified to harbor mesenchymal-epithelial transition factor (MET) gene amplification and neurotrophic tyrosine receptor kinase 1 (NTRK1) gene co-occurring mutation by targeted next-generation sequencing analysis upon the progression of empirical chemotherapy. He was then treated with a standard dose of crizotinib (250 mg, twice daily), which exhibited a satisfactory complete response (CR) of the targeted lesions after 1 month of treatment. When the number of renal cysts increased and renal inadequacy occurred after treatment for 2 months, crizotinib was reduced to half-dose (250 $\mathrm{mg}$, once daily), and still conferred maintenance of CR for another 6.5 months and good quality life of the patient. These results suggested that treatments based on driver genes rather than primary tumor types could be a promising manipulation for achieving better treatment outcome, and a half-dose of crizotinib might be both effective and tolerable for MET-overexpressed CUPs with underlying renal diseases.
\end{abstract}

Keywords: carcinoma of unknown primary, crizotinib, MET amplification, NTRK1 mutation, renal cyst

\section{Introduction}

Carcinomas of unknown primary (CUPs) are a heterogeneous group of cancers for which the metastases are clinically and histologically confirmed, but the primary tumor site remains occult after comprehensive workup. ${ }^{1}$ Patients with CUPs mainly received empirical chemotherapy (paclitaxel/platinum was the most commonly used regimen), and the median overall survival (OS) was within 1 year. ${ }^{2,3}$ In the era of precision medicine, the discovery of driver genes and the development of target agents enormously improve the survival and quality of life of patients with cancer. Although most malignant tumors are still treated according to the pathological classification of primary tumors, a growing number of studies have revealed that different malignant tumors with the same driver genes can be treated with the same targeted agents with good therapeutic effects. ${ }^{4}$ Therefore, the target treatment pattern according to driver genes but not primary tumor site may greatly improve 
the diagnosis and treatment of CUPs. In this study, the therapeutic efficiency of crizotinib was examined in a patient with CUP harboring mesenchymal-epithelial transition factor (MET) gene amplification and neurotrophic tyrosine receptor kinase 1 (NTRK1) gene cooccurring mutation and a history of long-term hypertension and renal cysts. The treatment strategy of CUPs based on molecular diagnosis, therapeutic effect of crizotinib, and management of related adverse events (AEs) was also discussed.

\section{Case report}

A 67-year-old male patient had a history of smoking with a smoking index of 800 . He had quit smoking for more than 1 year. He had a long-term history of hypertension and cerebral infarction. He regularly took his antihypertensive drugs, and his blood pressure was controlled. In January 2017, the patient accidentally found a mass in the right neck, accompanied by swelling and discomfort in the right upper extremity, which was not taken seriously at the beginning. Later, masses occurred in the right axillary fossa in succession. The patient was treated in a local hospital in April 2017. Enhanced neck and thoracic computed tomography (CT) showed 1) multiple enlarged lymph nodes (the maximum was $3.2 \times 2.4 \mathrm{~cm}$ ) in the right neck, right supraclavicular region, right axillary fossa, and right tracheal and esophageal sulcus and 2) nodule in the posterior segment of right upper lung $(0.63 \times 0.78 \mathrm{~cm})$. Pathological puncture of the right supraclavicular lymph node showed metastatic, poorly differentiated adenocarcinoma. Positron emission tomography (PET)/CT did not find a clear primary tumor, and the clinical diagnosis was "metastatic poorly differentiated adenocarcinoma with unknown primary site." The patient then received 6 cycles of TP (taxanes/cisplatin) regimen in the hospital. Partial remission (PR) of target lesions was assessed after 3 cycles of chemotherapy, and no significant change in the nodules in the right upper lung was seen. One month after the last chemotherapy, the lymph nodes in the right neck and right axillary region showed a progressive increase, indicating progression of the disease.

In October 2017, the patient was transferred to our department for treatment. The Eastern Cooperative Oncology Group (ECOG) score was 2 points. The patient was fatigued and had poor appetite. He lost $5 \mathrm{~kg}$ of weight. Physical examination revealed signs of anemia and multiple enlarged lymph nodes in the right neck and right axilla, with the largest one fused into a hard, painless mass, about $3 \mathrm{~cm}$ in diameter, with poor mobility. No obvious positive signs were found in other systems. PET/CT showed 1) multiple nodular soft tissue density shadows in the right neck, right upper and lower clavicular regions, right axilla and mediastinum, besides increased fluorodeoxyglucose (FDG) metabolism (SUVmax =10.8), which was considered as metastatic lymph nodes (Figure 1A); 2) nodular shadows in the posterior segment of the right upper lung, with not-so-high FDG metabolism (Figure 1B); and 3) multiple cerebral infarctions, splenomegaly, and multiple cysts in the left kidney. Laboratory tests indicated normal liver and kidney function; normal white blood cell, neutrophil, and platelet count; hemoglobin $87 \mathrm{~g} / \mathrm{L}$; and serum CEA $41.35 \mathrm{ng} / \mathrm{mL}$ (normal, $<5 \mathrm{ng} / \mathrm{mL}$ ). Clinical diagnosis indicated 1) metastatic adenocarcinoma in the right neck, right clavicular region, right axillary fossa, and mediastinal lymph nodes with unknown primary site (stage IV); 2) anemia (moderate); 3) hypertension of grade 2 (very high-risk group); 4) multiple cerebral infarctions; and 5) multiple cysts in the left kidney. The patient was poorly tolerant to further chemotherapy and had a willingness to seek targeted therapy. Thus, a second biopsy of the lymph nodes in the right neck was performed to obtain real-time pathology of the tumor tissue. Moreover, peripheral blood and freshly punctured tumor tissues were taken to perform the next-generation sequencing (NGS)-based multigene panel analysis (Burning Rock Biotech, Guangzhou, China) to reveal possible drug targets. The results of histopathological and immunohistochemical (IHC) diagnoses were consistent with those of metastatic poorly differentiated adenocarcinoma (Figure 1C-E): CK7 (2+), TTF-1 (-), CK5/ $6(-)$, and p63 (-). The targeted NGS analysis of neck metastatic carcinoma revealed EGFR mutation (-), ALK fusion $(-)$, ROS1 fusion (-), BRAF mutation (-), RAS mutation (-), MET gene amplification (9.12 times) (Figure 2A), NTRK1 gene c.339-2A > C mutation $(+)$ [at a mutant allele frequency (MAF) of 16.17\%] (Figure 2B), Cyclin D1 (CCND1) gene amplification (4.75 times) (Figure 2A), and TP53 gene R249S mutation (at a MAF of $11.54 \%$ ). Tumor mutation burden was moderate. The tumor was microsatellite stable. However, targeted NGS of ctDNA only identified NTRK1 (at a MAF of $0.42 \%$ ) (Figure 2B) and TP53 gene mutation (at a MAF of $0.39 \%$ ). IHC was performed against MET to verify the findings of the gene test, and the results indicated that the protein staining was positive and the expression level was high $(2+$ to $3+)$ (Figure 2C,D). Anti-MET-amplification therapy with crizotinib ( $250 \mathrm{mg}$, twice daily) was recommended in November 2017 on the basis of the molecular findings, 

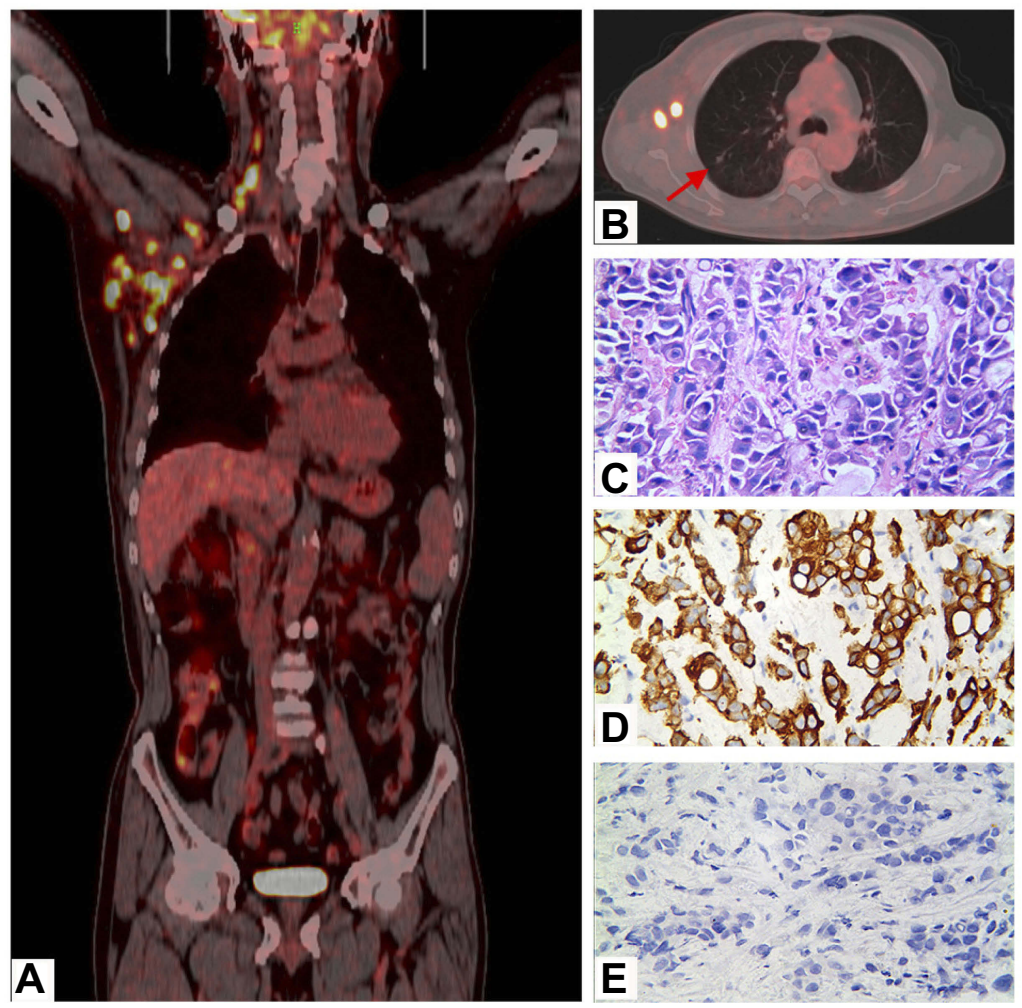

Figure I Imaging and histopathological characteristics of the patient before crizotinib treatment.

(A and B) PET/CT scans showed increased FDG uptake in multiple lymphatic metastases in the whole body and no FDG uptake in the small nodule located in the posterior segment of the right upper lung. (C) H\&E staining confirmed the right neck lymph nodes as poorly differentiated metastatic carcinomas. (D and E) Immunohistochemical staining of the right neck lymph nodes showed positive staining of CK7 $(2+)$ and negative staining of TTF-I $(\times 400)$.

Note: The red arrow in figure $B$ indicates the small nodule located in the right upper lung.

Abbrevations: CK7, cytokeratin 7; FDG, fluorodeoxyglucose; H\&E, hematoxylin \& eosin; PET/CT, positron emission tomography/computed tomography; TTF-I, thyroid transcription factor-I.

accessible drugs in China, and treatment willingness of the patient. After 1 week of medication, the patient's clinical symptoms ameliorated significantly and promptly. Scans demonstrated a complete response (CR) after 1 month of treatment (Figure 3), and the tumor markers returned to normal levels. During the administration of crizotinib, the main AEs were grade II creatinine elevation, grade I localized edema, and a new cyst in the right kidney (Figure 4). Considering the decrease in creatinine clearance and long-term history of chronic hypertension, the dose was reduced by half ( $250 \mathrm{mg}$, once daily) for maintenance after 2 months of medication, and treatments for protecting the kidneys were combined. Tumor was excellently controlled until the patient died of tumor progression in September 2018, with a progression-free survival (PFS) and OS time of 8.5 months and 10.0 months, respectively, from the initiation of crizotinib. The treatment using halfdose crizotinib was well tolerated, with a good quality of life and without deterioration in renal complications.

\section{Discussion Exploration of precise treatment mode for CUPs}

As one of the characteristics of malignant tumors, genomic instability and mutation were important links in the occurrence and development of tumors. ${ }^{5}$ The rapid development of molecular biology and translational medicine led to the discovery of different tumor-driven genes using highthroughput NGS to assist the judgment of primary tumors and guidance of treatment. ${ }^{6-8}$ It may bring new enlightenment to the treatment of CUPs because if this treatment mode is verified, the CUPs may be the optimal indication for targeted therapy based on genetic information regardless of tumor location. For example, Ross et al found that gene mutation was most commonly observed in adenocarcinoma among the 200 samples of CUPs, and $72 \%$ of adenocarcinoma with an unknown primary site had mutations of RTK and Ras pathways. ${ }^{9}$ Gatalica et al also obtained similar conclusions by analyzing 1,086 CUPs 

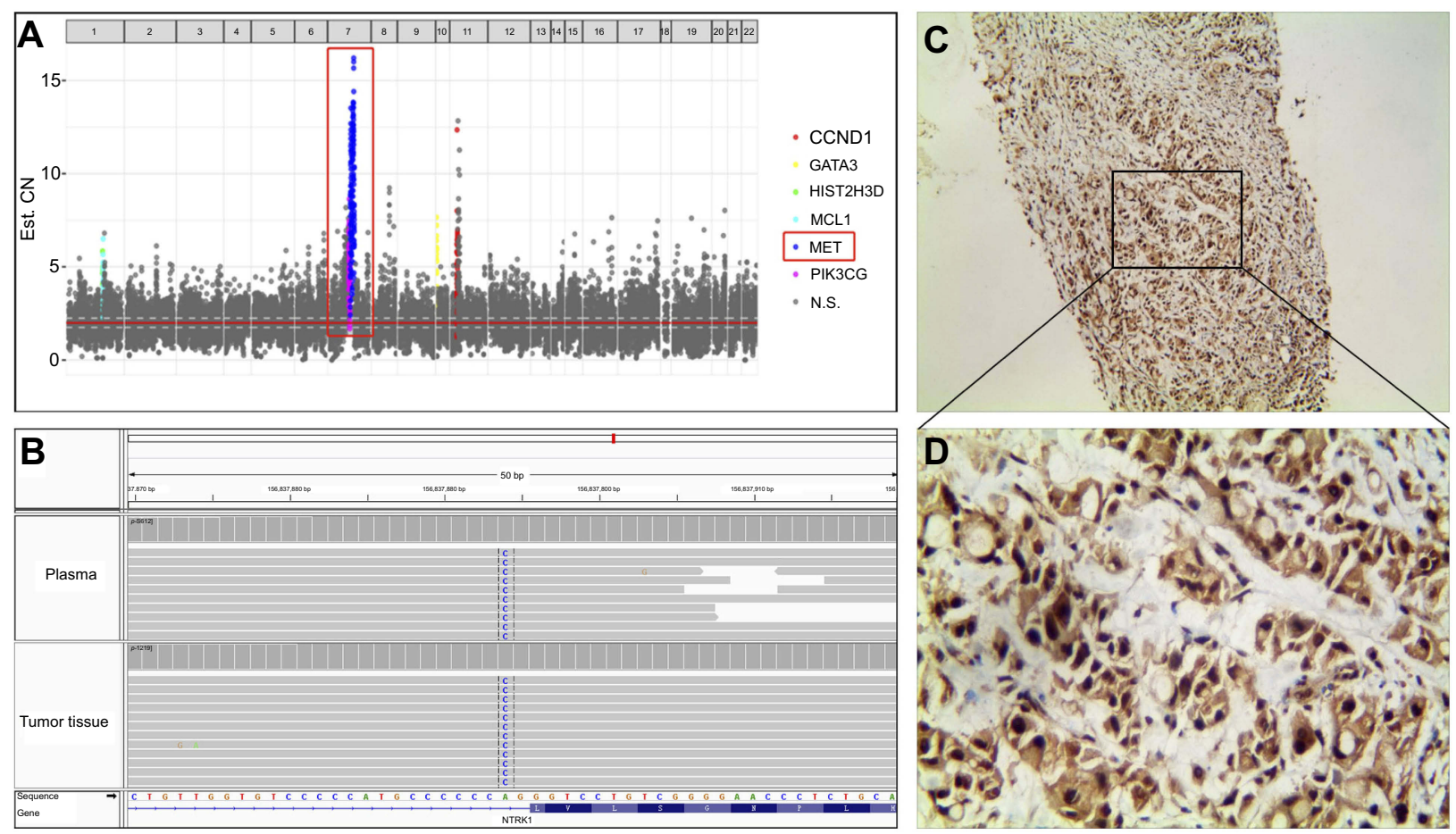

Figure 2 Molecular analysis of the patient before crizotinib treatment.

(A) NGS analysis based on tissue samples showed MET and CCNDI copy-number amplification. (B) NGS analysis based on plasma samples showed NTRKIc.339-2 A>C mutation. (C and D) IHC staining of tissue samples showed positive staining of MET protein (2 to $3+, \times 100$, and 400 , respectively) in metastatic lesions.

Abbrevations: CCNDI, cyclin DI; GATA3, GATA binding protein 3; HIST2H3D, histone cluster 2 H3D; IHC, immunohistochemistry; MCLI, myeloid cell leukemia I; MET, mesenchymal-epithelial transition factor; NGS, next-generation sequencing; N.S., no significance; NTRKI, neurotrophic tyrosine receptor kinase I; PI3KCG, phosphatidylinositol 3-kinase catalytic subunit gamma.

and concluded that the expression profiling analysis of tumor genes could provide more treatment options for patients with CUPs. ${ }^{10}$

The present case was of an elderly male with a metastatic adenocarcinoma of unknown primary. He received the empirical chemotherapeutic TP regimen and obtained a first-line PFS of less than 6 months. His quality of life drastically deteriorated, although the target lesion was temporarily and partially relieved. After the patient was admitted to our department, we confirmed his diagnosis and revealed a MET gene amplification of the patient as evidenced by both NGS and IHC analyses of the tumor tissues. The patient gained his first $\mathrm{CR}$ of the target lesions after 1 month of treatment with anti-MET-amplification drug crizotinib, and the CR was maintained for another 6.5 months in the case of subsequent medication with half the dose. The good effect of crizotinib in treating this patient was speculated to have come from precise gene profiling and effective drug use, but the possibility that it might be related to the unique biological behavior of the CUPs themselves cannot be ruled out. In the meantime, it was unknown whether other composite mutations in this patient also influenced this process because tumors harboring NTRK1 fusion, such as MPRIP-NTRK1 and LMNANTRK1, might be sensitive to crizotinib treatment. ${ }^{11,12}$ Indeed, c.339-2A $>C$ variable splicing mutation on the sixth exon of NTRK1, which was located in the leucinerich repeat region, was identified in this patient. Because this kind of variation was not reported in the COSMIC and cBioPortal databases, the significance was unknown for this novel case report, and hence further research is warranted in the future.

\section{Treatment of crizotinib-related renal AEs}

According to the instructions for crizotinib, $4 \%$ of patients may have complicated renal cysts during medication, ${ }^{13}$ which has now increased to more than $22 \%{ }^{14,15}$ Related studies found that the use of crizotinib was associated with the growth of existing renal cysts and the development of new renal cysts. ${ }^{16}$ Renal cysts generally occur in the early stage of crizotinib treatment. The older the age, the higher the incidence, which can be complicated (Bosniak class III or higher) or be similar to malignancy. At the same time, previous studies also found that patients with significant 

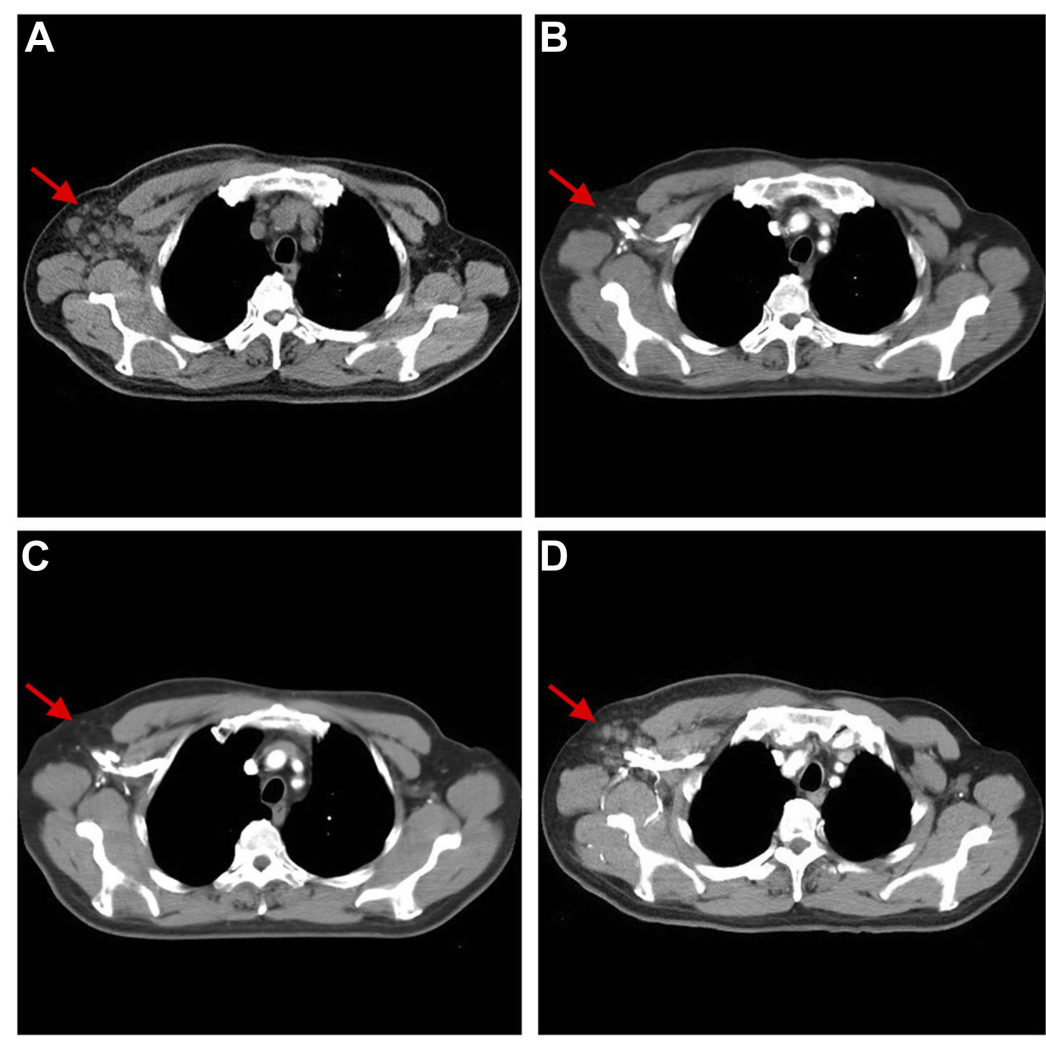

Figure 3 Chest CT scans showing dynamic changes in metastatic right axillary lymph nodes (A) before, (B) I month after, (C) 4 months after, and (D) 8.5 months after crizotinib treatment.

Note: The red arrows in all figure parts indicate the metastatic lesions located in the right axillary fossa.

Abbrevation: $\mathrm{CT}$, computed tomography.
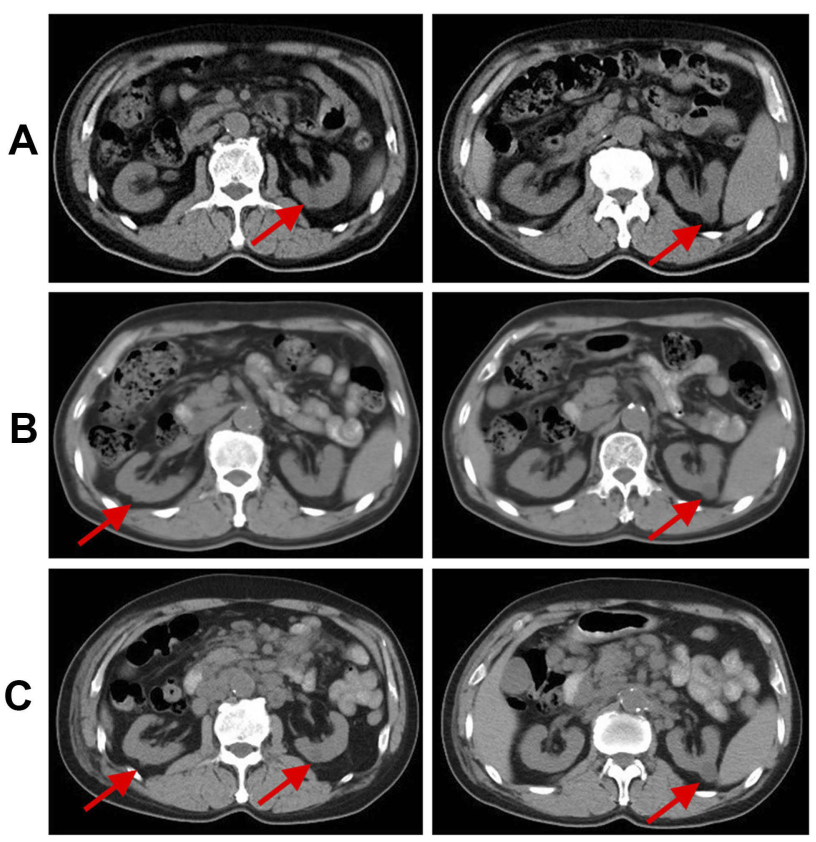

Figure 4 Abdominal CT scans showing dynamic changes in renal cysts $(\mathbf{A})$ before, (B) I month after, and (C) 8.5 months after crizotinib treatment.

Note: The red arrows in all figure parts indicate the cysts located in both kidneys.

Abbrevation: $\mathrm{CT}$, computed tomography. changes in renal cysts usually received more antitumor treatment before crizotinib was used and also received crizotinib for a longer duration. The current consensus was that the occurrence of renal cysts after crizotinib treatment might be a benign process that could be spontaneously resolved by discontinuing the drug, and that it would not hinder the use of crizotinib. ${ }^{14,15,17}$ Although the mechanism of action of crizotinib in the formation of renal cysts was not fully understood, studies suggested that it might be associated with the inhibition of c-MET and activation of the NF- $\mathrm{kB}$ pathway, leading to renal injury and fibrosis. ${ }^{18}$

The present case was of an elderly patient with underlying hypertension. Unlike previous crizotinib-associated renal cysts in clinical trials, this patient not only experienced an increase in the number of left renal cysts but also had a new cyst in his right kidney, along with grade II creatinine elevation and grade I localized edema. The emergence and management of renal-related AEs in this patient would provide new insights into the use of crizotinib. Recent studies on crizotinib-related renal cysts were based mostly on retrospective studies of clinical trials for 
crizotinib, and in such population, patients often had no history of hypertension or kidney disease. Due to the strong renal compensatory capacity, the single population may mask the damage of renal cysts to renal function, resulting in research bias. The medical history and imaging data of this patient revealed that long-term chronic hypertension caused the renal cortex to become thinner, which was consistent with the imaging findings of hypertensive contracted kidney. Crizotinib might aggravate renal inadequacy that already existed and was unrecognized in the patient, causing deterioration of renal function, thereby eliciting the elevation of creatinine and occurrence of edema. Therefore, it is necessary for the clinicians to be aware of the potential complication of renal cysts caused by the use of crizotinib. Especially for patients with a history of long-term hypertension or kidney disease, regular monitoring of renal function, and reexamination of renal CT should be performed to avoid more severe renal impairments.

\section{Conclusion}

In summary, this study reported the clinical course of an elderly man with a metastatic adenocarcinoma. MET amplification and unclear NTRK1 mutations were identified using targeted NGS analysis upon the progression of empirical chemotherapy. The treatment with half the standard dose of crizotinib resulted in not only a satisfactory CR but also an effective management of drug-related renal AEs, in this CUP patient harboring MET amplification and NTRK1 co-occurring mutation. Therefore, gene profiling may contribute to the precise diagnosis and treatment of CUPs. The clinical management of targeted drugs is critically important in promoting the precision medicine, especially in drug-related AEs.

\section{Ethics approval and informed consent}

This study was approved by the ethics committee of the First Affiliated Hospital of Bengbu Medical College, and written informed consent to participate was obtained from the family member of the patient.

\section{Consent for publication}

Written informed consent was obtained from the family member of the patient for the publication of this case report and any accompanying images.

\section{Abbreviations}

AE, adverse event; CCND1, cyclin D1; CR, complete response; CT, computed tomography; CUP, carcinoma of unknown primary; ECOG, Eastern Cooperative Oncology Group; FDG, fluorodeoxyglucose; IHC, immunohistochemistry; MAF, mutant allele frequency; MET, mesenchymal-epithelial transition factor; NGS, next-generation sequencing; NTRK1, neurotrophic tyrosine receptor kinase 1; OS, overall survival; PET, positron emission tomography; PFS, progression-free survival; PR, partial remission; $\mathrm{TP}$, taxanes/cisplatin.

\section{Acknowledgments}

The authors sincerely thank the patient for his contribution to the publication of this case report. This work was supported by the Excellent Young Talents Fund Program of Higher Education Institutions of Anhui Province (No. gxfx2017066) and an internal grant from the First Affiliated Hospital of Bengbu Medical College (No. Byyfykj201801).

\section{Disclosure}

The authors report no conflicts of interest in this work.

\section{References}

1. Varadhachary GR, Raber MN. Cancer of unknown primary site. N Engl J Med. 2014;371(8):757-765. doi:10.1056/nejmra1303917

2. Petrakis D, Pentheroudakis G, Voulgaris E, Pavlidis N. Prognostication in cancer of unknown primary (CUP): development of a prognostic algorithm in 311 cases and review of the literature. Cancer Treat Rev. 2013;39(7):701-708. doi:10.1016/j.ctrv.2013.03.001

3. Bochtler T, Löffler H, Krämer A. Diagnosis and management of metastatic neoplasms with unknown primary. Semin Diagn Pathol. 2018;35(3):199-206. doi:10.1053/j.semdp.2017.11.013

4. Rashdan S, Gerber DE. Going into BATTLE: umbrella and basket clinical trials to accelerate the study of biomarker-based therapies. Ann Transl Med. 2016;4(24):529. doi:10.21037/atm.2016.04.05

5. Hanahan D, Weinberg RA. Hallmarks of cancer: the next generation. Cell. 2011;144(5):646-674. doi:10.1016/j.cell.2011.02.013

6. Hainsworth JD, Greco FA. Gene expression profiling in patients with carcinoma of unknown primary site: from translational research to standard of care. Virchows Arch. 2014;464(4):393-402. doi:10.1007/ s00428-014-1545-2

7. Greco FA. Molecular diagnosis of the tissue of origin in cancer of unknown primary site: useful in patient management. Curr Treat Options Oncol. 2013;14(4):634-642. doi:10.1007/s11864-013-0257-1

8. Varadhachary GR, Talantov D, Raber MN, et al. Molecular profiling of carcinoma of unknown primary and correlation with clinical evaluation. J Clin Oncol. 2008;26(27):4442-4448. doi:10.1200/ JCO.2007.14.4378

9. Ross JS, Wang K, Gay L, et al. Comprehensive genomic profiling of carcinoma of unknown primary site: new routes to targeted therapies. JAMA Oncol. 2015;1(1):40-49. doi:10.1001/jamaoncol.2014.216

10. Gatalica Z, Millis SZ, Vranic S, et al. Comprehensive tumor profiling identifies numerous biomarkers of drug response in cancers of unknown primary site: analysis of 1806 cases. Oncotarget. 2014;5 (23):12440-12447. doi:10.18632/oncotarget.2574 
11. Zhou N, Schäfer R, Li T, Fang M, Liu L. A primary undifferentiated pleomorphic sarcoma of the lumbosacral region harboring a LMNA-NTRK1 gene fusion with durable clinical response to crizotinib: a case report. BMC Cancer. 2018;18(1):842. doi:10.1186/ s12885-018-4242-8

12. Vaishnavi A, Capelletti M, Le AT, et al. Oncogenic and drug-sensitive NTRK1 rearrangements in lung cancer. Nat Med. 2013;19(11):1469-1472. doi:10.1038/nm.3352

13. FDA Published Crizotinib Label. Available from: http://www.access data.fda.gov/drugsatfda_docs/label/2013/202570s006lbl.pdf. Accessed October 27, 2018.

14. Lin Y-T, Wang Y-F, Yang JC-H, et al. Development of renal cysts after crizotinib treatment in advanced ALK-positive non-small-cell lung cancer. J Thorac Oncol. 2014;9(11):1720-1725. doi:10.1097/ JTO.0000000000000326
15. Schnell P, Bartlett $\mathrm{CH}$, Solomon BJ, et al. Complex renal cysts associated with crizotinib treatment. Cancer Med. 2015;4 (6):887-896. doi:10.1002/cam4.437

16. Halpenny DF, McEvoy S, Li A, et al. Renal cyst formation in patients treated with crizotinib for non-small cell lung cancer-Incidence, radiological features and clinical characteristics. Lung Cancer. 2017;106:33-36. doi:10.1016/j.lungcan.2017.01.010

17. Cameron LB, Jiang DHS, Moodie K, Mitchell C, Solomon B, Parameswaran BK. Crizotinib Associated Renal Cysts [CARCs]: incidence and patterns of evolution. Cancer Imaging. 2017;17(1):7. doi:10.1186/s40644-017-0109-5

18. Yasuma T, Kobayashi T, D‘Alessandro-Gabazza CN, et al. Renal injury during long-term crizotinib therapy. Int J Mol Sci. 2018;19 (10). doi:10.3390/ijms19102902

\section{Publish your work in this journal}

OncoTargets and Therapy is an international, peer-reviewed, open access journal focusing on the pathological basis of all cancers, potential targets for therapy and treatment protocols employed to improve the management of cancer patients. The journal also focuses on the impact of management programs and new therapeutic agents and protocols on patient perspectives such as quality of life, adherence and satisfaction. The manuscript management system is completely online and includes a very quick and fair peer-review system, which is all easy to use. Visit http://www.dovepress.com/ testimonials.php to read real quotes from published authors. 